\title{
桥连对嵌段共聚物自组装的调控
}

\author{
李卫华* \\ (复旦大学聚合物分子工程国家重点实验室＼cjkstart高分子科学系上海 200438)
}

\begin{abstract}
摘要 通过嵌段共聚物自组装形成 “桥连” 是制备具有优异力学性能的网络结构的有效途经, 具有重要的应用价值. 但 是, 过去的研究工作很少讨论 “桥连” 对嵌段共聚物自组装行为本身的影响. 该研究评论主要总结了最近几年利用 “桥 连” 对嵌段自组装行为进行调控的工作进展. 作者设计了 $\mathrm{BABCB}$ 三组分线性多嵌段共聚物，当其自组装形成二元 “介 观晶体” (球、柱)结构时，中间 B 嵌段连接 $\mathrm{A}$ 和 C 相区(嵌段聚集成的 “大原子”), 自然地形成桥连; 减小中间桥连 B 嵌段的相对长度, 就可以增加其拉伸程度, 从而降低介观晶体的配位数; 另外, 两个末端 $\mathrm{B}$ 嵌段的相对长度可以直接调 控 $\mathrm{A}$ 和 $\mathrm{C}$ “大原子”之间的相对配位数. 基于这两个机理, 自洽场理论计算预测了各种配位数相等和不相等的二元介 观晶体结构. 进一步, 将 “拉伸桥连” 概念拓展到 $\mathrm{AB}$ 型嵌段共聚物体系中, 并且通过多臂星型嵌段共聚物分子结构中 的 “组合构型摘效应” 在 $\mathrm{AB}$ 型嵌段共聚物中形成高比率的桥连构型, 使传统的六角柱状结构转变为了四配位的四方 柱状和三配位的石墨烯类柱状结构. 未来, 在 $\mathrm{ABC}$ 三组分嵌段共聚物体系的设计中引入拓扑结构以及使用共混等方 法, 有望在介观尺度重铸大多数已知的原子/离子二元晶体结构, 甚至超越原子/离子晶体结构.
\end{abstract}

关键词＼cjkstart桥连；嵌段共聚物；自组装；介观晶体；自洽场理论

\section{"Bridge" Makes Differences to the Self-assembly of Block Copolymers}

\author{
Weihua Li*
}

(State Key Laboratory of Molecular Engineering of Polymers, Department of Macromolecular Science, Fudan University, Shanghai 200438, China)

\begin{abstract}
To form "bridge" via the self-assembly of block copolymer provides a useful way for the fabrication of network structures of excellent mechanical properties, which is promising in applications. However, previous work has hardly paid attention to the impact of "bridge" on the self-assembly behavior of block copolymers. This account provides a review of a recent progress about the control of the self-assembly behaviors of block copolymers via the stretching degree of the bridging block. Accordingly, we have purposely designed BABCB linear multiblock copolymer. When BABCB copolymer self-assembles into binary mesocrystal structures (sphere or cylinder), the middle B-block connects a pair of A and C domains ("macromolecular atom" aggregated by blocks) naturally forming bridge. The stretching degree of the middle bridging B-block can be increased by reducing its length relative to the other two B-blocks, lowering the coordination numbers (CNs) of mesocrystal. Moreover, the asymmetry of CNs between A and C "macromolecular atoms" can be tuned by the asymmetry between the two end B-blocks. Abiding by the two principles, using self-consistent field theory (SCFT) we have predicted rich binary mesocrystals of equal and unequal CNs. Furthermore, we have extent the concept of "stretched bridge" into AB-type block copolymers. We have proposed the effect of combinatorial entropy to realize high-ratio bridging configurations in the self-assembled structures by AB-type block copolymers. By increasing the stretching degree of bridging blocks, we have successfully predicted nonclassical square array and graphene-like array of cylinders instead of the usual hexagonal array of cylinders. In future, it is hopeful to recast most of known atomic/ionic binary crystal structures or even beyond by considering topology and blending during the design of ABC-type block copolymers.

Keywords bridge; block copolymer; self-assembly; mesocrystal; self-consistent field theory
\end{abstract}

\section{1 引言}

嵌段共聚物是由不同化学成分的聚合物通过共价 键连接形成的, 不同嵌段之间的不相容性使它们倾向于 发生相分离，而牢固的共价键连接将相分离限制在分子 链尺度上. 因此，嵌段共聚物会自发地发生微观相分离， 被称为自组装. 嵌段共聚物自组装可以形成周期性有序
结构，结构的周期通常在 $10 \sim 200 \mathrm{~nm} ，$ 可以用于制备高 密度存储器、多孔材料、光子晶体等 ${ }^{[1-4]}$. 在过去几十年 里, 嵌段共聚物自组装一直吸引了大量的实验和理论研 究兴趣 ${ }^{[5-8]}$. 随着合成方法的不断发展, 实验上合成的嵌 段共聚物分子的种类越来越多, 包含各种链拓扑结构 (线型、星型、梳型、瓶一刷型等), 形成越来越丰富的有

*E-mail: weihuali@fudan.edu.cn; Tel.: 021-31243579

Received September 22, 2020; published November 9, 2020.

Project supported by the National Natural Science Foundation of China (No. 21925301).

项目受国家自然科学基金(No. 21925301)资助. 
序纳米结构. 同时, 各种研究嵌段共聚物自组装的理论 方法也被逐渐发展起来. 其中最为成功的一个方法是自 洽场理论 ${ }^{[9-10]}$, 它有几个显著的优点: (1)可以精确地计 算各种有序结构的自由能, 从而确定其热力学稳定性; (2) 可以计算自由能的界面能贡献和熵贡献, 以及每一 个链段的空间分布, 这些结果有利于深入理解嵌段共聚 物的自组装机理; (3)适用范围广, 不仅可以考虑各种链 拓扑结构, 还可以计算熔体、溶液、几何受限以及共混 体系; (4)已经发展了多个数值求解方法 ${ }^{[11-13]}$, 其中准谱 方法是目前最被广泛使用的方法之一, 它不仅效率高, 而且适用的体系多. 最近几年, 我们在前人的工作 ${ }^{[13-14]}$ 基础上, 进一步发展了求解自洽场理论的准谱方法. 一 方面, 发展了场的特殊初始化方法, 解决了复杂结构的 求解难题 ${ }^{[15]}$; 另一方面, 利用结构空间对称性对准谱方 法进行了加速 ${ }^{[16]}$. 高效精确的自洽场计算使我们可以 开展系统深入的理论研究, 不仅加深了对实验结果的理 解 ${ }^{[17-22]}$, 所预测的新结果还促进了新的实验研究 ${ }^{[23-33]}$. 因此, 实验和理论的相互促进将嵌段共聚物自组装的研 究不断向前推进.

无论是实验还是理论, 以往的研究主要聚焦于探索 某一给定嵌段共聚物的自组装行为, 例如有序无序相变 和所形成的有序结构, 是一个典型的正向问题的求解, 发现新结构的效率很低 ${ }^{[34]}$. 最近几年, 我们提出 “根据 目标结构, 反向推演嵌段共聚物分子结构和组成” 的理 念 ${ }^{[15]}$. 根据已知嵌段共聚物的自组装机理, 我们建立了 一系列嵌段共聚物分子结构设计的指导原理 ${ }^{[15,24,27,35-38]}$, 其中一个最重要的原理就是 “拉伸桥连” ${ }^{[15]}$. 依据这些 原理, 我们针对很多新颖的有序结构, 甚至是非传统结 构, 成功设计出了相应的嵌段共聚物体系.

在嵌段共聚物自组装的研究中, “桥连” 并不是一 个新概念. 形成桥连的最简单的一个嵌段共聚物体系是 ABA 线性三嵌段共聚物, 在其分相结构中通常同时存 在环形和桥连构型, 其中桥连构型跨越两个相邻的相 区. 早在 20 多年前, 理论就计算了桥连的比率 ${ }^{[39]}$. 在 $\mathrm{ABAB} \ldots$ 多嵌段共聚物中, 随着嵌段数的增加, 桥连会 跨越更多的相区. 桥连连接多个相区的特性可以显著提 高结构的力学性能, 具有重要的应用价值. 例如聚苯乙 烯-聚丁二烯-聚苯乙烯(SBS)热塑性弹性体就是利用了 中间聚丁二烯嵌段的桥连形成网络结构, 从而获得优异 的力学性能. 此外, 很多实验研究表明多嵌段共聚物的 桥连作用可以大幅提高分相材料的韧性 ${ }^{[40-41]}$. 但是, 过 去的研究工作几乎没有讨论如果利用桥连调控嵌段共 聚物的自组装行为.

\section{2 “拉伸桥连效应” 对 $A B C$-型嵌段共聚物形成 二元介观晶体结构的调控}

$\mathrm{ABC}$ 线性三嵌段共聚物是最简单的以及实验和理 论研究最多的一种三组分嵌段共聚物. 决定 $\mathrm{ABC}$ 嵌段
共聚物自组装行为的参数至少 5 个: 三个相互作用参数 $\left(\chi_{\mathrm{AB}} N, \chi_{\mathrm{BC}} N, \chi_{\mathrm{AC}} N\right)$ 和两个独立的体积分数(例如: $\left.f_{\mathrm{A}}, f_{\mathrm{B}}\right)$, 因此它可能形成的有序结构的种类异常丰富 ${ }^{[40]}$. 但是, 当给定三个嵌段的化学成分, 也就是固定了三个相互作 用参数之间的相对强度, 所能形成的有序结构的种类依 然较少 ${ }^{[42-43]}$. 例如, 对于 $\chi_{\mathrm{AB}} N=\chi_{\mathrm{BC}} N=\chi_{\mathrm{AC}} N$ 以及 $f_{\mathrm{A}}=f_{\mathrm{C}}$, 所能形成的二元球状相是体心排列的, 和二元离子晶体 $\mathrm{CsCl}$ 的晶格类似, 因此我们把它称为 $\mathrm{CsCl}$-类型的二元 介观晶体, 把 $\mathrm{A}$ 和 $\mathrm{C}$ 嵌段聚集形成的球状相区看成是人 造 “大分子原子” ( “Macromolecular atoms”, MAs), 简 称“大原子”. 显然, $\mathrm{A}$ 和 $\mathrm{C}$ 大原子的配位数(coordination number, $\mathrm{CN}$ )均为 8 . 我们知道, 二元离子晶体的种类非 常丰富. 即使碱金属和卤族原子构成的二元离子晶体, 除了 $\mathrm{CsCl}$-类型, 还有 $\mathrm{NaCl}$-类型, 其配位数是 $\mathrm{CN}=6$. 那么, 为什么 $\mathrm{ABC}$ 三嵌段共聚物形成的二元介观晶体 不排列成 $\mathrm{NaCl}$-类型的晶格? 另外, 我们能否通过 $\mathrm{ABC}$-类型的嵌段共聚物形成更多种类的晶体结构? 对 于聚合物, 最重要的一个变量是其链结构. 因此, 从链 结构的设计出发实现各种二元介观晶体结构是一个可 行的思路.

在 $\mathrm{ABC}$ 三嵌段共聚物形成的二元介观晶体中，对 于每一根嵌段共聚物链, 其 $\mathrm{A}$ 和 $\mathrm{C}$ 嵌段分别位于 $\mathrm{A}$ 和 $\mathrm{C}$ 大原子中, 那么中间的 $\mathrm{B}$ 嵌段自然地形成桥连，连接每 一对 $\mathrm{A} / \mathrm{C}$ 大原子, 也就是说桥连的比率接近 $100 \%$, 和 $\mathrm{ABA}$ 体系中的部分桥连不同. 为了清楚起见, 我们给出 了二维的示意图(图 1), 红色和蓝色的实心圆分别代表 $\mathrm{A}$ 和 $\mathrm{C}$ 大原子. 但是, 由于 $\mathrm{B}$ 嵌段必须均匀地填充整个 基体，它需要通过形成一个大的环去填充最远端; 换句 话说, 它的拉伸程度不可以自由地调节. 为了调节桥连 $\mathrm{B}$ 嵌段的拉伸程度, 我们必须引入新的变量, 改变 $\mathrm{B}$ 嵌 段填充基体的方式. 最简单的方法就是引入多个 $\mathrm{B}$ 嵌 段，使得基体的部分空间可以由非桥连的 $\mathrm{B}$ 嵌段进行填 充，从而使桥连嵌段的拉伸程度可以自由地调节.

显然, 存在很多种增加 $\mathrm{B}$ 嵌段的方式(嵌段数和连 接方式), 换句话说, 可选择的链结构非常多, 通过简单 的试错, 很难找到目标分子结构. 我们通过分析二元介 观晶体的形成机理, 找到了一种通过链结构调控其配位 数的有效方法. 图 1 展示了相关的机理: 左边是 $\mathrm{CN}=4$ 的四方排列的二元晶格, 右边是 $\mathrm{CN}=3$ 石墨烯类型的 晶格. 为了简单起见, 我们假定 $\mathrm{A}$ 和 $\mathrm{C}$ 圆盘的尺寸相等 且恒定. 如果两种晶格的 $\mathrm{A} / \mathrm{C}$ 盘子之间的距离相等, 那 么左边晶格中每个盘子所对应的空白面积就比右边的 小. 要保持两边空白面积所占比率相等, 就需要减小右 边晶格中 $\mathrm{A} / \mathrm{C}$ 盘子的距离, 也就是使左边的晶格距离大 于右边的. 这是一个非常重要的结论, 该结论可以在 $\mathrm{ABC}$-型嵌段共聚物自组装结构中实现.

我们知道对称的 $\mathrm{ABC}$ 三嵌段共聚物自组装所形成 的二元柱状结构排列成图 1 中右边的晶格. 我们如果缩 
短桥连的 B-嵌段, 并且把所减少的 B 嵌段连接到 $\mathrm{A}$ 和 $\mathrm{C}$ 嵌段的末端, 保持 $\mathrm{B}$ 嵌段的体积守恒; 那么, 就可以通 过中间 $\mathrm{B}$-桥连的拉伸减小相邻 $\mathrm{A} / \mathrm{C}$ 盘子之间的距离, 也 就是缩小晶格距离. 但是为了保持体积守恒, $\mathrm{A} / \mathrm{C}$ 盘子 的周期性排列必须发生改变, 向更加稀疏的低配位晶格 转变, 也就是左边的三配位晶格. 这就是拉伸桥连调控 介观晶体结构的基本机理: 在 BABCB 多嵌段共聚物自 组装中, 减小中间 B-嵌段的相对长度, 增加 B-桥连的拉 伸, 就会减小晶格距离, 从而迫使晶体结构向低配位的 晶格转变 ${ }^{[13]}$.

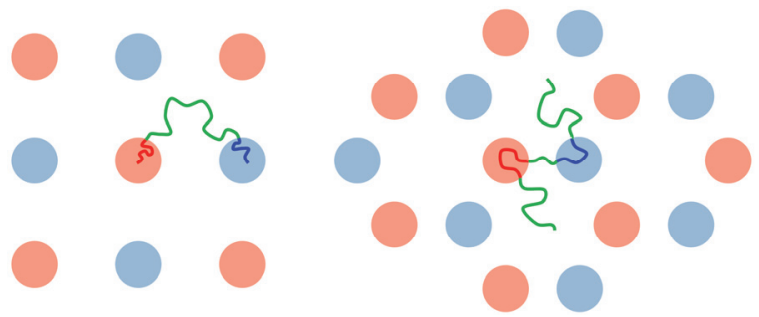

图 1 拉伸桥连对 $\mathrm{ABC}$-型嵌段共聚物形成二元介观晶体结构的调控 机理: 左图给出了 $\mathrm{ABC}$ 线性三嵌段共聚物在四方二元介观晶体中的 典型链构型, 中间 B 嵌段形成松弛的桥连; 右图给出了 BABCB 五嵌 段共聚物在三配位二元介观晶体中的链构型, 中间 B 桥连嵌段被显著 拉伸.

Figure 1 Schematics demonstrating the effect of stretching bridging block on the formation of binary mesocrystals from the self-assembly of ABC-type block copolymers. The left panel presents a typical chain configuration of $\mathrm{ABC}$ linear triblock copolymer in the tetragonal binary mesocrystal, where the middle bridging B-block forms a large loop. The right panel shows a typical configuration of BABCB pentablock copolymer in the three-coordinated mesocrystal, where the middle bridging B-block is highly stretched.

通过自洽场理论计算, 比较不同晶格结构的自由 能, 就可以验证上述机理. 我们首先考虑 $\mathrm{B}_{1} \mathrm{AB}_{2} \mathrm{CB}_{3}$ 的 一个简单情况: 固定 $\chi_{\mathrm{AB}} N=\chi_{\mathrm{BC}} N=\chi_{\mathrm{AC}} N=80, f_{\mathrm{A}}=f_{\mathrm{C}}=f$, $f_{\mathrm{B} 1}=f_{\mathrm{B} 3}$ 和 $f_{\mathrm{B} 2}=1-2 f_{\mathrm{B} 1}-2 f$. 由于该分子结构是对称的, 也就是 $\mathrm{A}$ 和 $\mathrm{C}$ 等价的, 因此它所形成的晶体结构中 $\mathrm{A}$ 和 $\mathrm{C}$ 大原子的配位数相等. 在图 2 中, 我们比较了 $\mathrm{CsCl}$ $(\mathrm{CN}=8), \mathrm{NaCl}(\mathrm{CN}=6)$ 和 $\mathrm{ZnS}(\mathrm{CN}=4)$ 三种介观晶体的 自由能, 发现随着中间嵌段的相对长度 $f_{\mathrm{B} 2}$ 的减小, $\mathrm{CsCl}$ 依次转变成了 $\mathrm{NaCl}$ 和 $\mathrm{ZnS}^{[13]}$. 继续减小 $f_{\mathrm{B} 2}, \mathrm{ZnS}$ 会继续 转变为更低配位数的晶体结构(例如 $\alpha-\mathrm{BN}, \mathrm{CN}=3$ ).

除了配位数相等的二元晶体结构, 还存在很多配位 数不相等的二元晶体结构. 只要将多嵌段共聚物的分子 结构从对称变为不对称, 我们就可以获得配位数不相等 的二元介观晶体, 其平均配位数依然可以通过调节桥连 的拉伸程度进行调控. 最简单的一种非对称的 $\mathrm{ABC}$-类 型分子是 $\mathrm{AB}_{2} \mathrm{CB}_{3}\left(f_{\mathrm{B} 1}=0\right)$. 当 $f_{\mathrm{A}}=f_{\mathrm{C}}$ 且 $\mathrm{A}$ 和 $\mathrm{C}$ 嵌段形成 同种几何形状(球或者柱)的相区(即大原子)时, 由于 $\mathrm{A}$ 的自由末端可以停留在大原子内任意位置, 而 $\mathrm{C}$ 嵌段和 两个 $\mathrm{B}$ 嵌段的连接点则必须位于大原子的表面上(如图 3), 为了减小 $\mathrm{C}$ 嵌段的拉伸, 所形成的 $\mathrm{C}$ 大原子必须比
A 大原子小. 由于两种大原子的总体积相同，所以 $\mathrm{C}$ 大 原子的个数比 $\mathrm{A}$ 大原子的个数多, 也就是 $\mathrm{C}$ 的配位数比 $\mathrm{A}$ 的配位数低.
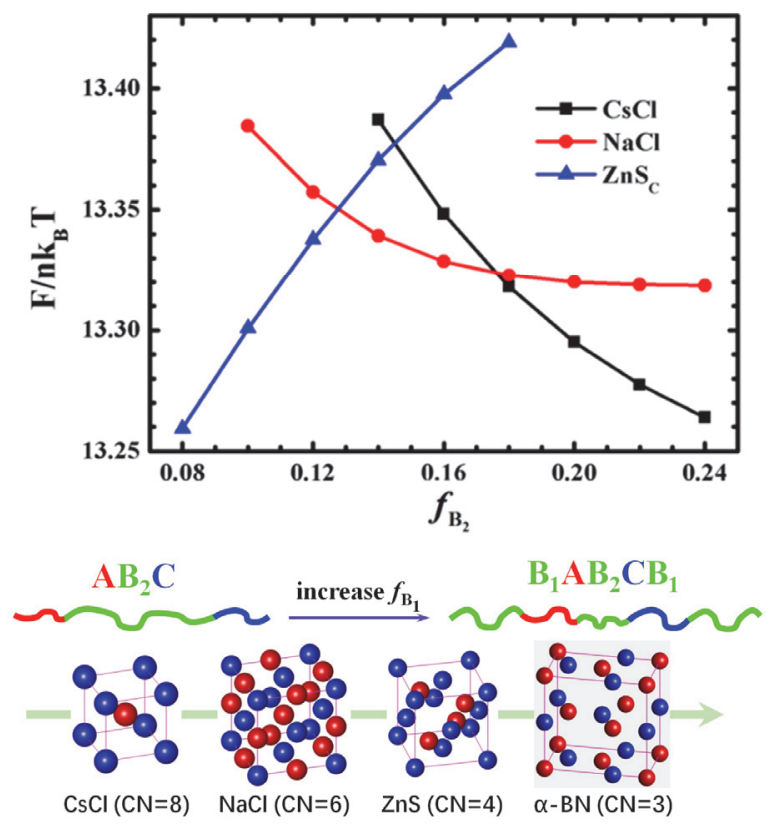

图 $2 \mathrm{BABCB}$ 嵌段共聚物所形成的不同二元介观晶体的自由能比较 以及相应的结构转变序列: $\chi_{\mathrm{AB}} N=\chi_{\mathrm{BC}} N=\chi_{\mathrm{AC}} N=80, f_{\mathrm{A}}=f_{\mathrm{C}}=f$ 和 $f_{\mathrm{B} 1}=$ $f_{\mathrm{B} 3}$.

Figure 2 Free-energy comparison as well as the transition sequence of different mesocrystals in BABCB block copolymers with $\chi_{\mathrm{AB}} N=\chi_{\mathrm{BC}} N=$ $\chi_{\mathrm{AC}} N=80, f_{\mathrm{A}}=f_{\mathrm{C}}=f$ and $f_{\mathrm{B} 1}=f_{\mathrm{B} 3}$ (Reproduced from Ref. [15]. Copyright 2020. American Chemical Society).

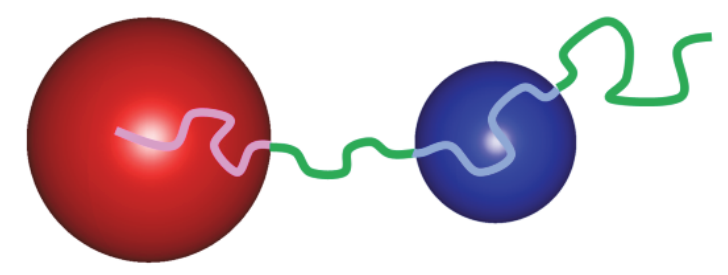

图 $3 \mathrm{ABCB}$ 嵌段共聚物在 $\chi_{\mathrm{AB}} N=\chi_{\mathrm{BC}} N=\chi_{\mathrm{AC}} N$ 和 $f_{\mathrm{A}}=f_{\mathrm{C}}$ 条件下形成 的 $\mathrm{A}$ 球(红色)比 $\mathrm{C}$ 球(蓝色)大的示意图.

Figure 3 Schematics illustrating that A-sphere (red) is larger than $\mathrm{C}$-sphere (blue) in the binary mesocrystal formed by $\mathrm{ABCB}$ tetrablock copolymer with $\chi_{\mathrm{AB}} N=\chi_{\mathrm{BC}} N=\chi_{\mathrm{AC}} N$ and $f_{\mathrm{A}}=f_{\mathrm{C}}$.

图 4 给出了自洽场理论计算所构建的 $\mathrm{A} \mathrm{B}_{2} \mathrm{CB}_{3}$ 关于 $f$ 和 $f_{\mathrm{B} 2}$ 的相图以及相应的结构图. 这个相图包含了多个 配位数不相等的二元介观晶体: $\mathrm{AlB}_{2}, \mathrm{Li}_{3} \mathrm{Bi}, \mathrm{CaF}_{2}, \mathrm{Nb}_{3} \mathrm{Sn}$ (A15), $\mathrm{TiO}_{2}, \mathrm{ReO}_{3}$ 以及一些二维的二元介观晶体. 显然, 这些结构的平均配位数是随着 $f_{\mathrm{B} 2}$ 减小而下降的, 因此 依然遵循桥连拉伸对配位数的调控规则. 如果, 从对称 的 $\mathrm{B}_{1} \mathrm{AB}_{2} \mathrm{CB}_{3}$ 连续变化到非对称的 $\mathrm{AB}_{2} \mathrm{CB}_{3}$, 二元介观 晶体的转变过程中配位数的不对称性也会逐渐增大, 我 们观察到配位数从 $1: 1$ 变为 $2: 3 、 1: 2$ 以及 $1: 3$ (图 5) ${ }^{[15]}$. 


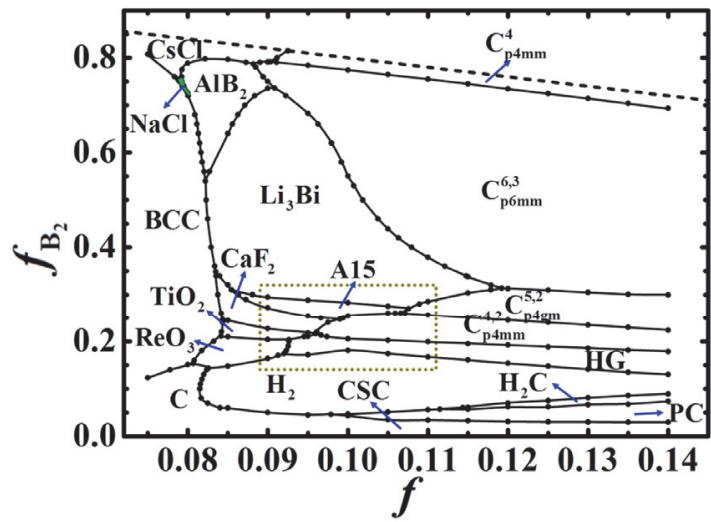

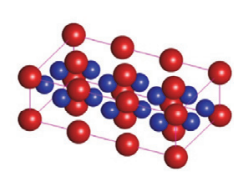

$\mathrm{AlB}_{2}(\overline{\mathrm{CN}}=8)$

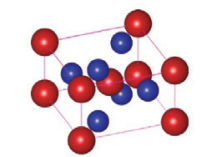

$\mathrm{TiO}_{2}(\overline{\mathrm{CN}}=4)$

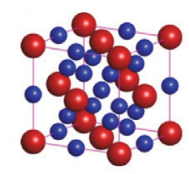

$\mathrm{Li}_{3} \mathrm{Bi}(\overline{\mathrm{CN}}=7)$

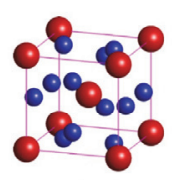

$\mathbf{A 1 5}(\overline{\mathrm{CN}}=6)$

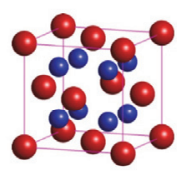

$\mathrm{CaF}_{2}(\overline{\mathrm{CN}}=16 / 3)$
图 $4 \chi_{\mathrm{AB}} N=\chi_{\mathrm{BC}} N=\chi_{\mathrm{AC}} N=80$ 和 $f_{\mathrm{A}}=f_{\mathrm{C}}=f$ 时 $\mathrm{AB}_{2} \mathrm{CB}_{3}$ 四嵌段共聚物关 于 $f$ 和 $f_{\mathrm{B} 2}$ 的相图以及一些典型有序结构的示意图.

Figure 4 Phase diagram with respect to $f$ and $f_{\mathrm{B} 2}$ of $\mathrm{AB}_{2} \mathrm{CB}_{3}$ tetrablock copolymer with $\chi_{\mathrm{AB}} N=\chi_{\mathrm{BC}} N=\chi_{\mathrm{AC}} N=80$ and $f_{\mathrm{A}}=f_{\mathrm{C}}=f$, as well as the schematic plots for some typical ordered structures (Reproduced from Ref. [15]. Copyright 2020. American Chemical Society).

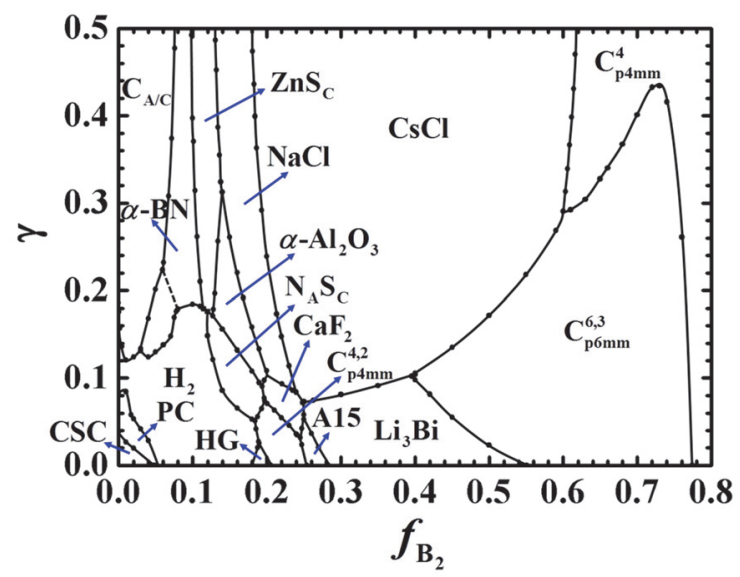

图 $5 \chi_{\mathrm{AB}} N=\chi_{\mathrm{BC}} N=\chi_{\mathrm{AC}} N=80$ 和 $f_{\mathrm{A}}=f_{\mathrm{C}}=f$ 时 $\mathrm{B}_{1} \mathrm{AB}_{2} \mathrm{CB}_{3}$ 嵌段共聚物关 于 $f_{\mathrm{B} 2}$ 和 $\gamma=f_{\mathrm{B} 1} / f_{\mathrm{B} 3}$ 的相图.

Figure 5 Phase diagram with respect to $f_{\mathrm{B} 2}$ and $\gamma=f_{\mathrm{B} 1} / f_{\mathrm{B} 3}$ of $\mathrm{B}_{1} \mathrm{AB}_{2} \mathrm{CB}_{3}$ block copolymer with $\chi_{\mathrm{AB}} N=\chi_{\mathrm{BC}} N=\chi_{\mathrm{AC}} N=80$ and $f_{\mathrm{A}}=f_{\mathrm{C}}=f$ (Reproduced from Ref. [15]. Copyright 2020. American Chemical Society).

\section{3 “拉伸桥连效应” 对 AB-型多嵌段共聚物自 组装的调控}

$\mathrm{ABC}$-型嵌段共聚物自组装可以形成二元介观晶体, 总是存在桥连 $B$-嵌段连接一对 $A / C$ 大原子. 而对于 $A B-$ 型嵌段共聚物, 其小组分嵌段(例如 $\mathrm{A}$ 嵌段)可以聚集形
成一种大原子，这些单一种类大原子在大组分基体中周 期性排列成单元介观晶体. 很多研究表明, AB-型嵌段 共聚物形成的单元介观晶体主要为经典的体心立方球 状结构 $(\mathrm{BCC}, \mathrm{CN}=12.5)$ 和六角排列的柱状结构 $(\mathrm{CN}=$ 6) ${ }^{[6]}$. 如果我们可以在单元介观晶体中构建桥连, 并且 对其拉伸进行调节, 就可以获得不同配位数的单元介观 晶体，例如更低配位的面心立方结构( $\mathrm{FCC}, \mathrm{CN}=12.5)$ 、 四方排列的柱状结构 $(\mathrm{CN}=4)$.

最关键的一个问题是如何在同种大原子之间实现 高比率的桥连. 我们之前已经提到: AB-型嵌段共聚物 也可以形成桥连, 如 ABA 三嵌段共聚物(如图 6); 但是 桥连构型的比率通常不够高, 在球状结构中是 $75 \%$ $80 \%$, 而在柱状结构中只有 $60 \% \sim 65 \%{ }^{[39]}$. 我们提出: $(\mathrm{AB})_{n}$ 星型结构的 “组合构象熵效应” 可以大幅度提高 桥连的比率, 例如当臂数 $n=5$ 时, 球状结构的桥连比率 超过了 $99 \%$ ，而柱状结构的也超过了 $95 \%{ }^{[44]}$. 实现了高 比率的桥连后, 我们需要解决的问题就是调节桥连的拉 伸程度. 为此, 我们设计了两种分子结构(图 7). 在分子 结构 M1 中，是通过引入一个均聚物臂实现对桥连相对
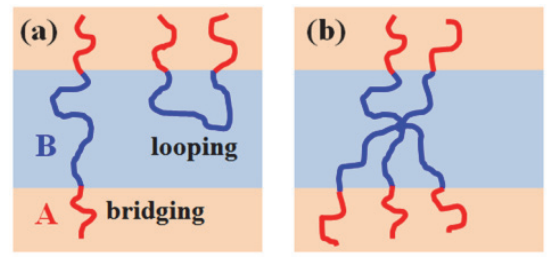

(c)

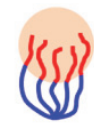

图 6 线性 $\mathrm{ABA}$ 和星型 $(\mathrm{AB}){ }_{n}$ 嵌段共聚物链结构形成桥连或环构型的 示意图.

Figure 6 Schematics illustrating the bridging or looping configuration formed by ABA linear and (AB) $)_{n}$ star block copolymer architectures (Reproduced from Ref. [36]. Copyright 2020. American Chemical Society).

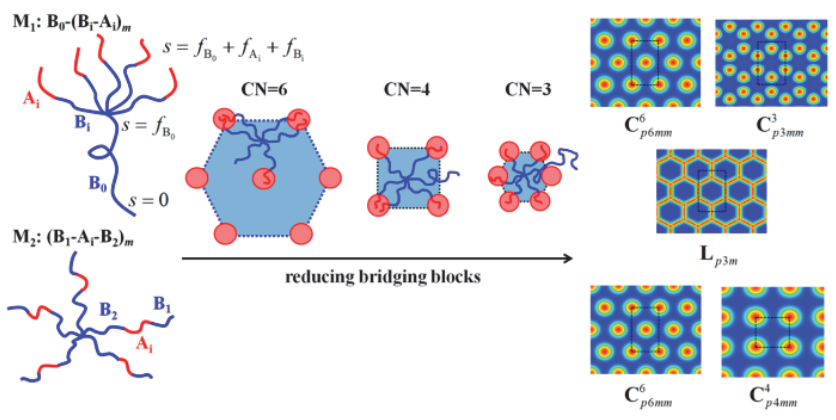

图 7 左边: 所设计的两个嵌段共聚物分子的结构示意图; 中间: 三 个配位数不同的柱状结构中链构型的示意图, 展示了拉伸桥连效应对 晶格排列的影响; 右边: 所设计的嵌段共聚物自组装形成的稳定结构 的密度分布图.

Figure 7 Left: schematic plot of the architectures of two designed AB-type multiblock copolymers, M1 and M2. Center: illustrative plot of chain configurations in three typical cylindrical morphologies with different coordination numbers demonstrating the formation of bridges among neighboring domains and their impact on the domain arrangement (i.e., packing lattice). Right: density profiles of the stable morphologies self-assembled from the two block copolymers (Reproduced from Ref. [35]. Copyright 2020. American Physical Society). 
长度的改变; 而在分子结构 $\mathrm{M} 2$ 中, 是将 $\mathrm{AB}$ 两嵌段变为 了 $\mathrm{BAB}$ 三嵌段, 这样就可以对中心的桥连 $\mathrm{B}_{2}$-嵌段的相 对长度进行改变. 最终, 自洽场理论成功预测了稳定的 非经典四方柱状结构、三配位的柱状结构和 FCC 球状 结构 ${ }^{[35]}$.

\section{4 “拉伸桥连效应” 和其他效应的协同作用}

除了 “拉伸桥连效应”，最近几年我们还提出了其 他调控嵌段共聚物自组装行为的效应: “局域分凝效 应” ${ }^{[27,37]}$ 和 “堆积受挫缓解效应” ${ }^{[38]}$, 这些效应构成了 一套嵌段共聚物分子结构设计的指导规则. 我们发现: 如果所设计的分子可以同时实现多个效应, 就可以获得 更加丰富的介观晶体结构. 例如, 最近我们设计了一种 $\mathrm{AB}-$ 型多嵌段共聚物, 同时实现了拉伸桥连效应和堆积 受挫缓解效应(图 8) ${ }^{[45]}$. 在所设计的嵌段共聚物分子结 构中, 桥连是由 $\mathrm{B}_{2}$ 嵌段形成的. 由于堆积受挫主要来源 于 $\mathrm{B}$ 嵌段均匀填充基体时的非均匀拉伸, 因此我们设计 了两种不同长度的 $\mathrm{B}_{1}$ 和 $\mathrm{B}_{3}$ 嵌段去填充基体: 长的嵌段 填充远的空间, 而短的嵌段填充近的空间; 从而充分缓 解了 $\mathrm{B}$ 嵌段的堆积受挫. 在该嵌段共聚物自组装结构 中, 虽然桥连的比率不是非常高, 但是拉伸桥连效应和 堆积受挫缓解效应的协同作用增强了对介观晶体结构 的调控效果, 成功导致了多个新颖的非传统介观晶体的 形成, 除了前面提到的 FCC 结构、四方柱状结构和三配 位的柱状结构, 还有立方金刚石球状结构 $\left(\mathrm{DS}_{\mathrm{C}}, \mathrm{CN}=\right.$ 4)、简单立方球状结构 $(\mathrm{SC}, \mathrm{CN}=6)$ 、独特的六角对位层 排列的球状结构 $\left(\mathrm{iHP}^{\mathrm{a}}, 6<\mathrm{CN}<8\right)$ 以及矩形柱状结构 $\left(\mathrm{C}_{\text {rect }}, 2<\mathrm{CN}<4\right)$.

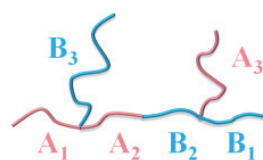

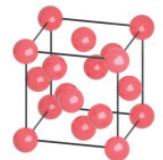

$\mathbf{D S}_{\mathrm{C}}$

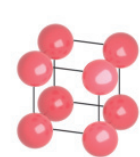

SC

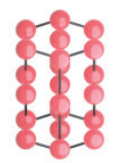

iHPa
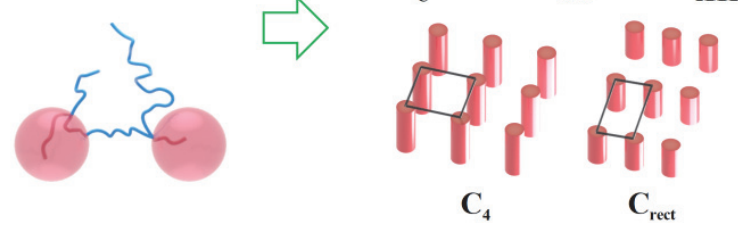

图 8 在所设计的 $\mathrm{AB}$-型嵌段共聚物自组装结构中, 同时实现了 “拉 伸桥连效应” 和 “堆积受挫缓解效应” ; 这两个效应的协同作用导致 了多个新颖的低配位单元介观晶体结构的形成. 需要指出的是: $\mathrm{B}_{2}$ 嵌 段除了可以形成桥连, 也可以形成环(loop), 也就是 $\mathrm{A}_{1} / \mathrm{A}_{2}$ 和 $\mathrm{A}_{3}$ 嵌段位 于同一个 $\mathrm{A}$ 相区内, 但是该示意图没有给出这种构型.

Figure 8 "Effect of stretched bridge" and "effect of released packing frustration" are realized simultaneously in the ordered morphologies self-assembled by a purposely designed AB-type block copolymer melt, leading to the formation of a number of low-coordinated single mesocrystal structures. It is necessary to point out that $\mathrm{B}_{2}$-block can also forming a looping configuration in addition to the bridging one, that is, $\mathrm{A}_{1} / \mathrm{A}_{2}$ and $A_{3}$-blocks are located within the same A-domain. The looping configuration of $\mathrm{B}_{2}$-block is not shown in the schematic (Reproduced from Ref. [45]. Copyright 2020. American Chemical Society).

\section{5 结论与展望}

本研究评论简要总结了我们课题组在过去几年里 关于 “拉伸桥连效应对嵌段共聚物自组装调控” 的工作. 在 $\mathrm{BABCB}$ 嵌段共聚物体系中, 通过调节中间桥连嵌段 的拉伸程度, 成功调控了自组装形成的二元介观晶体结 构的配位数, 从而获得了丰富的二元介观晶体结构, 包 括配位数相等和不相等的. 然后，通过 “组合构象熵效 应”，在 $\mathrm{AB}$-型嵌段共聚物中实现了高比率的桥连嵌段 以及对桥连的拉伸进行了调节，从而获得了各种新颖的 低配位的单元介观晶体结构. 值得指出的是, 我们的很 多理论结果已经得到了实验的证实 ${ }^{[30-31,46-48]}$. 例如, Miyamori 等 ${ }^{[48]}$ 在 ABAC 体系中观察了类十二轴准晶结 构, “拉伸桥连效应” 对该结构的形成起到了关键作用, 证实了我们的理论预测 ${ }^{[25]}$. 一方面, 我们的工作加深了 对嵌段共聚物自组装机理的认识; 另一方面，丰富了嵌 段共聚物自组装的结构库，拓宽了嵌段共聚物自组装的 应用，为嵌段共聚物分子结构的设计打开了一扇窗户。

一般来说, 分子结构决定凝聚态结构, 凝聚态结构 决定性能. 因此，根据凝聚态结构，反向推演嵌段共聚 物分子结构对于聚合物分相材料的设计具有重要的意 义. 目前, 我们所考虑的链拓扑结构还非常有限, 特别 是对于 $\mathrm{ABC}$-型的嵌段共聚物分子, 只考虑了线性链. 在未来, 在 $\mathrm{ABC}$-型嵌段共聚物分子的设计中, 引入各 种链拓扑结构, 有希望在介观尺度重铸大多数原子/离 子晶体结构, 甚至获得超越原子/离子晶体结构的、新颖 的介观晶体结构. 此外, 在低配位的介观晶体结构中, 桥连嵌段被额外地拉伸, 一定会影响材料的性能. 因此, 对相关介观晶体的力学性能的研究, 无论对于理论还是 实验都是非常重要的课题.

\section{作者简介}

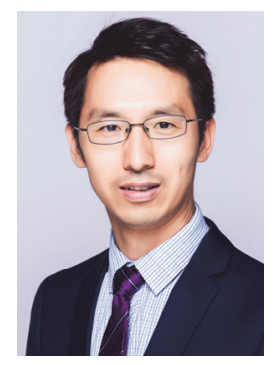

李卫华, 复旦大学高分子科学系教授, 1999、2004 年在上 海交通大学物理系获得学士、博士学位. 2004 2007 年在加拿 大 St. Francis Xavier 大学和 McMaster 大学从事博士后研究. 2007 年加入复旦大学聚合物分子工程国家重点实验室.

\section{References}

[1] Bates, F. S.; Fredrickson, G. H. Ann. Rev. Phys. Chem. 1990, 41, 525.

[2] Hamley, I. W. Prog. Polym. Sci. 2007, 32, 1152.

[3] Kim, H. C.; Park, S. M.; Hingsberg, W. D. Chem. Rev. 2010, 110, 146. 
[4] Li, W. H.; Müller, M. Prog. Polym. Sci. 2016, 54-55, 47.

[5] Bates, F. S.; Hillmyer, M. A.; Lodge, T. P.; Bates, C. M.; Delaney, K. T.; Fredrickson, G. H. Science 2012, 336, 434.

[6] Matsen, M. W. Macromolecules 2012, 45, 2161.

[7] Liu, D.; Wang, Y. Y.; Sun, Y. C.; Han, Y. Y.; Cui, J.; Jiang, W. Chin. J. Polym. Sci. 2018, 36, 888 .

[8] Liu, K.; Yang, C. M.; Yang, B. M.; Zhang, L.; Huang, W. C.; Ouyang, X. P.; Qi, F. G.; Zhao, N.; Bian, F. G. Chin. J. Polym. Sci. 2020, 38,92 .

[9] Shi, A. C. Development in Block Copolymer Science and Technology, Wiley, New York, 2004.

[10] Fredrickson, G. H. The Equilibrium Theory of Inhomogeneous Polymers, Oxford University Press, Oxford, 2006.

[11] Matsen, M. W.; Schick, M. Phys. Rev. Lett. 1994, 72, 2660.

[12] Drolet, F.; Fredrickson, G. H. Phys. Rev. Lett. 1999, 83, 4317.

[13] Tzeremes, G.; Rasmussen, K. Ø.; Lookman, T.; Saxena, A. Phys. Rev. E 2002, 65, 041806.

[14] Rasmussen, K. Ø.; Kalosakas, G. J. Polym. Sci. Part B: Polym. Phys. 2002, 40, 1777.

[15] Xie, N.; Liu, M. J.; Deng, H. L.; Li, W. H.; Qiu, F.; Shi, A. C. J. Am. Chem. Soc. 2014, 136, 2974.

[16] Qiang, Y. C.; Li, W. H. Macromolecules 2020, 53, 9943.

[17] Xu, Y. C.; Li, W. H.; Qiu, F.; Yang, Y. L.; Shi, A. C. J. Phys. Chem. $B$ 2010, 114, 14875.

[18] Li, W. H.; Qiu, F.; Shi, A. C. Macromolecules 2012, 45, 503.

[19] Liu, M. J.; Li, W. H.; Qiu, F.; Shi, A. C. Macromolecules 2012, 45, 9522.

[20] Xie, N.; Li, W. H.; Qiu, F.; Shi, A. C. ACS Macro Lett. 2014, 3, 906.

[21] Xia, B. K.; Li, W. H.; Qiu, F. Acta Chim. Sinica 2014, 72, 30 (in Chinese). (夏涁凯, 李卫华, 邱枫, 化学学报, 2014, 72, 30.)

[22] Xia, Y. M.; Li, W. H. Polymer 2019, 166, 21.

[23] Shao, Z. W.; Zhang, D.; Hu, W. G.; Xu, Y. C.; Li, W. H. Polymer 2019, 177, 202.

[24] Xie, N.; Liu, M. J.; Deng, H. L.; Li, W. H.; Qiu, F.; Shi, A. C. J. Am. Chem. Soc. 2014, 136, 2974.

[25] Duan, C.; Zhao, M. T.; Qiang, Y. C.; Chen, L.; Li, W. H.; Qiu, F.; Shi, A. C. Macromolecules 2018, 51, 7713.

[26] Miyamori, Y.; Suzuki, J.; Takano, A.; Matsushita, Y. ACS Macro
Lett. 2020, 9, 32.

[27] Liu, M. J.; Qiang, Y. C.; Li, W. H.; Qiu, F.; Shi, A. C. ACS Macro Lett. 2016, 5, 1167.

[28] Lindsay, A. P.; Lewis, R. M.; Lee, B.; Peterson, A. J.; Lodge, T. P.; Bates, F. S. ACS Macro Lett. 2020, 9, 197.

[29] Zhao, B.; Jiang, W. B.; Chen, L.; Li, W. H.; Qiu, F.; Shi, A. C. ACS Macro Lett. 2018, 7, 95.

[30] Ahn, S.; Kim, J. K.; Zhao, B.; Duan, C.; Li, W. H. Macromolecules 2018, 51, 4415.

[31] Ahn, S.; Seo, Y.; Kim, J. K.; Duan, C.; Zhang, L. X.; Li, W. H. Macromolecules 2019, 52, 9039.

[32] Zhao, M. T.; Li, W. H. Macromolecules 2019, 52, 1832.

[33] Müller, A. J.; Lindsay, A. P.; Jayaraman, A.; Lodge, T. P.; Mhanthappa, M. K.; Bates, F. S. ACS Macro Lett. 2020, 9, 576.

[34] Matsen, M. W. J. Phys.: Condens. Matter 2002, 14, R21.

[35] Gao, Y.; Deng, H. L.; Li, W. H.; Qiu, F.; Shi, A. C. Phys. Rev. Lett. 2016, 116, 068304.

[36] Li, W. H.; Duan, C.; Shi, A. C. ACS Macro Lett. 2017, 6, 1257.

[37] Jiang, W. B.; Qiang, Y. C.; Li, W. H.; Qiu, F.; Shi, A. C. Macromolecules 2018, 51, 1529.

[38] Xie, Q.; Qiang, Y. C.; Li, W. H. ACS Macro Lett. 2020, 9, 278.

[39] Matsen, M. W.; Thompson, R. B. J. Chem. Phys. 1999, 111, 7139.

[40] Hermel, T. J.; Hahn, S. F.; Chaffin, K. A.; Gerberich, W. W.; Bates, F. S. Macromolecules 2003, 36, 2190.

[41] Fleury, G.; Bates, F. S. Macromolecules 2009, 42, 3598.

[42] Bates, F. S.; Fredrickson, G. H. Phys. Today 1999, 52, 32.

[43] Qin, J.; Bates, F. S.; Morse, D. C. Macromolecules 2010, 43, 5128.

[44] Spencer, R. K. W.; Matsen, M. W. Macromolecules 2017, 50, 1681.

[45] Xie, Q.; Qiang, Y. C.; Chen, L.; Xia, Y. M.; Li, W. H. ACS Macro Lett. 2020, 9, 980 .

[46] Schulze, M. W.; Lewis, R. M.; Lettow, J. H.; Hickey, R. J.; Gillard, T. M.; Hillmyer, M. A.; Bates, F. S. Phys. Rev. Lett. 2017, 118, 207801.

[47] Lindsay, A. P.; Lewis, R. M.; Lee, B.; Peterson, A. J.; Lodge, T. P.; Bates, F. S. ACS Macro Lett. 2020, 9, 197.

[48] Miyamori, Y.; Suzuki, J.; Takano, A.; Matsushita, Y. ACS Macro Lett. 2020, 9, 32

(Cheng, B.) 